\title{
Score-based prediction of genomic islands in prokaryotic genomes using hidden Markov models Stephan Waack ${ }^{1}$, Oliver Keller ${ }^{1}$, Roman Asper ${ }^{1}$, Thomas Brodag1, Carsten Damm², Wolfgang Florian Fricke ${ }^{3}$, Katharina Surovcik ${ }^{1}$, Peter Meinicke ${ }^{4}$ and Rainer Merkl*5
}

\begin{abstract}
Address: ${ }^{1}$ Institut für Informatik, Universität Göttingen, Lotzestr. 16-18, 37083 Göttingen, Germany, ${ }^{2}$ Institut für Numerische und Angewandte Mathematik, Universität Göttingen, Lotzestr. 16-18, 37083 Göttingen, Germany, ${ }^{3}$ Göttingen Genomics Laboratory, Universität Göttingen, Grisebachstr. 8, 37077 Göttingen, Germany, ${ }^{4}$ Institut für Mikrobiologie und Genetik, Universität Göttingen, Goldschmidtstr. 1, 37077 Göttingen, Germany and ${ }^{5}$ Institut für Biophysik und Physikalische Biochemie, Universität Regensburg, Universitätsstr. 31, 93053 Regensburg, Germany

Email: Stephan Waack - waack@cs.uni-goettingen.de; Oliver Keller - keller@cs.uni-goettingen.de; Roman Asper - asper@cs.uni-goettingen.de; Thomas Brodag - Thomas.Brodag@T-Online.de; Carsten Damm - damm@math.uni-goettingen.de; Wolfgang Florian Fricke - wfricke@gwdg.de; Katharina Surovcik - surovcik@cs.uni-goettingen.de; Peter Meinicke - pmeinic@gwdg.de; Rainer Merkl*-Rainer.Merkl@biologie.uniregensburg.de

* Corresponding author
\end{abstract}

Published: 16 March 2006

BMC Bioinformatics 2006, 7:142 doi:10.1186/147/-2105-7-142
Received: 09 December 2005

Accepted: 16 March 2006

This article is available from: http://www.biomedcentral.com/I47/-2/05/7//42

(c) 2006 Waack et al; licensee BioMed Central Ltd.

This is an Open Access article distributed under the terms of the Creative Commons Attribution License (http://creativecommons.org/licenses/by/2.0), which permits unrestricted use, distribution, and reproduction in any medium, provided the original work is properly cited.

\begin{abstract}
Background: Horizontal gene transfer (HGT) is considered a strong evolutionary force shaping the content of microbial genomes in a substantial manner. It is the difference in speed enabling the rapid adaptation to changing environmental demands that distinguishes HGT from gene genesis, duplications or mutations. For a precise characterization, algorithms are needed that identify transfer events with high reliability. Frequently, the transferred pieces of DNA have a considerable length, comprise several genes and are called genomic islands (Gls) or more specifically pathogenicity or symbiotic islands.
\end{abstract}

Results: We have implemented the program SIGI-HMM that predicts Gls and the putative donor of each individual alien gene. It is based on the analysis of codon usage $(\mathrm{CU})$ of each individual gene of a genome under study. CU of each gene is compared against a carefully selected set of CU tables representing microbial donors or highly expressed genes. Multiple tests are used to identify putatively alien genes, to predict putative donors and to mask putatively highly expressed genes. Thus, we determine the states and emission probabilities of an inhomogeneous hidden Markov model working on gene level. For the transition probabilities, we draw upon classical test theory with the intention of integrating a sensitivity controller in a consistent manner. SIGI-HMM was written in JAVA and is publicly available. It accepts as input any file created according to the EMBL-format.

It generates output in the common GFF format readable for genome browsers. Benchmark tests showed that the output of SIGI-HMM is in agreement with known findings. Its predictions were both consistent with annotated Gls and with predictions generated by different methods.

Conclusion: SIGI-HMM is a sensitive tool for the identification of Gls in microbial genomes. It allows to interactively analyze genomes in detail and to generate or to test hypotheses about the origin of acquired genes. 


\section{Background}

Horizontal gene transfer (HGT) is a process that results in the acquisition of novel genes originating from perhaps taxonomically unrelated species. This phenomenon is frequent among microbes and is considered a means of rapid adaptation to changing environmental demands [1]. Pieces of DNA acquired via HGT frequently have a considerable length. These patches have been called genomic islands (GI) or due to their role and more specifically pathogenicity islands [2] or symbiotic islands [3].

Several methods have been developed for the prediction of GIs based on different approaches to identify putatively alien (pA) genes [4-12]. Each of these concepts has specific preferences and drawbacks; for recent reviews see $[13,14]$. In the following, we describe an approach which relies on the genome theory postulating a rather homogeneous codon usage within a genome [15]. The algorithm exploits taxon specific differences in codon usage for the identification of $\mathrm{pA}$ genes and the prediction of their putative origin. Hidden Markov models (HMMs) are a state of the art concept in computational learning theory. A sequence of observations is considered as being emitted from the states of an invisible Markov chain. The Viterbi algorithm efficiently computes a sequence of states that have the maximal posteriori probability given a certain sequence of observations and fixed transition and emission probabilities. The challenge in designing a HMM is representing the real situation adequately in order to generate relevant predictions. HMM have proved useful in many applications. In the case of predicting eukaryotic genes, for example, the programs GENSCAN $[16,17]$, HMMGene [18,19], GenomeScan [20], AUGUSTUS [21], and AUGUSTUS+ [22] are HMM-based.

It has been shown that HMMs allow to predict GIs [9]. GIs have typically a considerable length, therefore we have decided to implement a HMM assessing GI prediction on the gene level. GIs can originate from a variety of a priori unknown donors. Therefore, it is difficult to assure sufficient test statistics. We will describe an approach named SIGI-HMM. To some extent, it is based on principles introduced with SIGI [23]. This program was used to analyze individual genomes $[24,25]$ and to study the content of genomic islands in general [26] as well as to characterize gene-flux between bacteria and archaea [27]. For SIGIHMM we substituted a heuristic approach with a HMM. SIGI-HMM has only few parameters to adjust. The most relevant one is a sensitivity controller which affects transitions of the HMM in a consistent manner. We will demonstrate and assess the performance of SIGI-HMM by analyzing genomes in detail.

\section{Implementation}

We have implemented SIGI-HMM in Java as a first module of our software suite COLOMBO intended as a workbench for the statistical analysis of genomic data. The program can be downloaded from [28]. The download package contains also the program Artemis [29], which is used to visualize the output of SIGI-HMM. After the installation, a genomic dataset formatted in EMBL-format can be loaded and analyzed. SIGI-HMM creates several lists containing the predictions in GFF-format or tabulated. Predictions are classified according to the categories NATIVE and PUTAL. In addition, a modified EMBL-formatted file is generated containing both the original annotation and the predictions. This file can be fed into Artemis in order to color-code and visualize genome content. Thus, the user can interactively study the composition of genomes. Intentionally, only few parameters can be manipulated by the user: The sensitivity controller and the gap length which decides on merging single GIs to larger ones. In addition, the user can supplement the list of putative donors we have deduced from the CUTG database (see below). The default value of the the sensitivity controller was chosen to give predictions consistent with published results; see Table 1 . If it is known that the genome under study contains GIs, we propose the following approach in order to optimize sensitivity of SIGIHMM: Starting from a low value, sensitivity should be increased until all known GIs appear. If new islands emerge, they show the same degree of codon usage bias and should be considered GIs.

\section{Results}

The following text is organized as follows: First we introduce data models, the scoring system and the architecture of the HMM. Then we evaluate the predictive power of the algorithm and present analyses of several genomes.

\section{Stochastic data models}

Let $\mathcal{G}$ be a series of genes as deduced from a genome coding for proteins $\mathcal{P}$. For each codon $c$ we count its occurrence $\# c$ in $\mathcal{G}$. We define the synonymous frequency $q_{a c} \in$ $[0,1]$ as the ratio of $\# c$ divided by the occurrence of the amino acid $a$ encoded by $c$ in $\mathcal{P}$. The frequency $q_{c} \in[0,1]$ of $c$ in $\mathcal{G}$ is defined as $\# c$ divided by the occurrence of all codons in $\mathcal{P}$.

Now let $\mathcal{G}_{0}$ be a prokaryotic genome whose genomic islands have to be predicted and let $\mathcal{G}_{1}, \mathcal{G}_{2}, \ldots, \mathcal{G}_{r}$ be genomes assumed to be the donors for pA genes occurring in $\mathcal{G}_{0}$. We consider $\mathcal{G}_{1}$ to $\mathcal{G}_{r}$ as representatives of taxa $\mathcal{T}_{1}$ to $\mathcal{T}_{r}$ which are assumed to be the putative sources of $\mathcal{G}_{0}$ 's alien genes. For each protein (i.e. sequence of 
Table I: A comparison of pA predictions for prokaryotic species. SIGI-HMM was used to identify GIs. The accumulated length of genes constituting Gls is given in percent in column pA DNA. This transformation allows to compare results with entries of column Foreign DNA, which was reproduced from [4I]. The column Length lists the genome size im Mbp.

\begin{tabular}{lccc}
\hline Species & Length [Mbp] & Foreign DNA [\%] & PA DNA [\%] \\
\hline Escherichia coli K-I2 & 4.64 & 12.8 & 9.3 \\
Bacillus subtilis & 4.21 & 7.5 & 7.6 \\
Synechocystis PCC6803 & 3.57 & 16.6 & 5.0 \\
Deinococcus radiodurans & 2.65 & 5.2 & 4.8 \\
Archaeoglobus fulgidus & 2.18 & 5.2 & 4.2 \\
Aeropyrum pernix & 1.67 & 3.2 & 1.5 \\
Thermotoga maritima & 1.86 & 6.4 & 1.0 \\
Pyrococcus horikoshii & 1.74 & 2.7 & 2.9 \\
Methanobacterium thermoautotrophicum & 1.75 & 9.4 & 1.6 \\
Haemophilus influenzas Rd KW20 & 1.83 & 1.6 \\
Helicobacter pylori 26695 & 1.67 & 4.5 & \\
Aquifex aeolicus & 1.55 & 6.2 & 0.0 \\
Methanocaldococcus jannaschii & 1.66 & 9.6 & 1.8 \\
Treponema pallidum & 1.14 & 1.3 & 0.2 \\
Borrelia burgdorferi & 0.91 & 3.6 & 0.3 \\
Rickettsia prowazekii & 1.11 & 0.1 & 8.5 \\
Mycoplasma pneumoniae & 0.82 & 0.0 \\
Mycoplasma genitalium & 0.58 & 11.6 & 3.8 \\
\end{tabular}

amino acids) $\pi=a_{1}, a_{2}, \ldots, a_{n}$ that is encoded by a gene $g$ of genome $\mathcal{G}_{0}$ (given by the sequence of codons $c_{1}, c_{2}, \ldots, c_{n}$ ), and for each $\rho=0,1, \ldots, r$, we define the probability

$P_{\rho}(g \mid \pi):=q_{a_{1} c_{1}}^{(\rho)} \cdot q_{a_{2} c_{2}}^{(\rho)} \cdot \ldots \cdot q_{a_{n} c_{n}^{\prime}}^{(\rho)}$

where $q_{a c}^{(\rho)} \in[0,1]$ is the synonymous frequency in genome $\mathcal{G}_{\rho}$ as defined above.

\section{Scoring scheme}

We utilize the odds ratio

$$
\frac{P_{0}(g \mid \pi)}{P_{\rho}(g \mid \pi)}=\frac{q_{a_{1} c_{1}}^{(0)} \cdot q_{a_{2} c_{2}}^{(0)} \cdot \ldots \cdot q_{a_{n} c_{n}}^{(0)}}{q_{a_{1} c_{1}}^{(\rho)} \cdot q_{a_{2} c_{2}}^{(\rho)} \cdot \ldots \cdot q_{a_{n} c_{n}}^{(\rho)}}
$$

in the following way as a scoring scheme. The codon usage of $g$ originating from $\mathcal{G}_{0}$ resembles more the prevalences of $\mathcal{G}_{\rho}$ if

$$
\tau_{\rho, \alpha}>\frac{P_{0}(g \mid \pi)}{P_{\rho}(g \mid \pi)} .
$$

If this is the case for some $\rho$ and if

$$
\rho^{*}=\underset{\rho \in\{1,2, \ldots, r\}}{\arg \min } \frac{P_{0}(g \mid \pi)}{P_{\rho}(g \mid \pi)}
$$

then gene $g$ is considered to be pA originating from taxon $\mathcal{T}_{\rho^{*}}$ represented by genome $\mathcal{G}_{\rho^{*}}$. This principle of deducing the putative donor has previously been introduced and validated [23].

How to choose the thresholds $\tau_{\rho, \alpha}$ needed in Equation 2? Let $\mathcal{F}_{\pi}$ be the set of all theoretically possible genes coding for protein $\pi$. For each $\rho \in\{1,2, \ldots, r\}$, we consider the statistic

$t_{\rho}(G):=\ln \frac{P_{0}(G \mid \pi)}{P_{\rho}(G \mid \pi)}$

where $G \in \mathcal{F}_{\pi}$ is a random element distributed according to $P_{\rho}(\cdot \mid \pi)$. Having computed the mean $\mu_{\rho}$ and the standard deviation $\sigma_{\rho}$ of $t_{\rho}(G)$, we apply the central limit theorem: The random variable $1 / \sigma_{\rho}\left(t_{\rho}(G)-\mu_{\rho}\right)$ is approximately distributed according to the standard normal distribution with the cumulative distribution function $\Phi$. We determine the value $\tau_{\rho, \alpha}$ such that

$$
\alpha=1-\Phi\left(\frac{\ln \tau_{\rho, \alpha}-\mu_{\rho}}{\sigma_{\rho}}\right) .
$$

The parameter $\alpha$ serves as SIGI-HMM's sensitivity controller. It can be adjusted by the user. Please note that the 


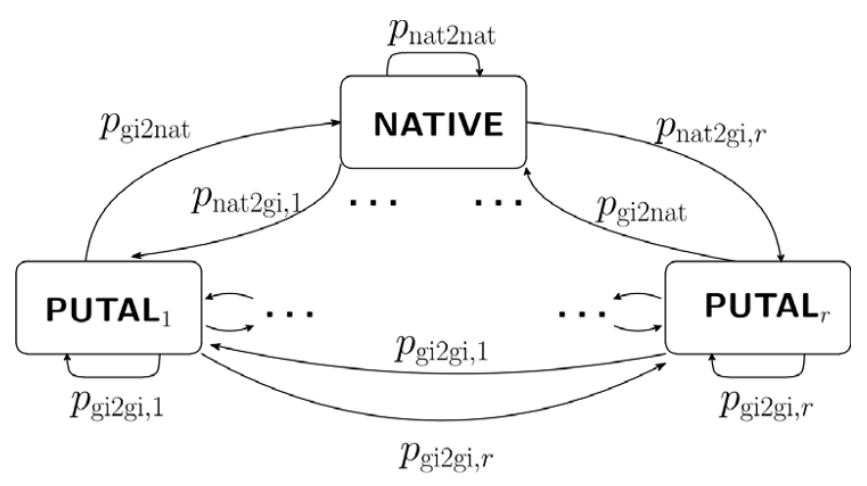

\section{Figure I}

States and transition probabilities of SIGI-HMM's Markov chain. The state NATIVE represents genes which are unsuspicious with respect to synonymous codon frequencies. For $\rho=1,2, \ldots, r$, the state $\mathrm{PUTAL}_{\rho}$ models genes, whose codon usage resembles more the prevalences of genomes $\mathcal{G}_{\rho}$ which represents taxon $\mathcal{T}_{\rho}$. Each transition from state $x$ to state $y$ is characterized by its transition probability $p_{x 2 y}$. In order to model the mosaic structure of $\mathrm{Gl}$ composition, transitions from any state $\mathrm{PUTAL}_{\rho}$ to any other one $\mathrm{PUTAL}_{\sigma}$ are allowed.

impact of parameter $\alpha$ onto the decision is independent of $\mathcal{G}_{0}$ and $\mathcal{G}_{\rho}$.

\section{Eliminating putatively highly expressed genes}

In several genomes, highly expressed genes show a specific codon usage which deviates from the average one and resembles codon prevalences observed in genes coding for ribosomal proteins; see e.g. [8]. We name these genes putatively highly expressed (PHX). On the one hand, it is unlikely that these genes were acquired via HGT. On the other hand, methods based on codon usage tend to classify them as pA. This needs to be prevented explicitly. We use an approach similar to the GCB score introduced in [30]. It was shown that this methods is one of the best to predict gene expressivity [31]. Let $q_{a c}^{(0, \text { rib) }}$ be the synonymous codon frequencies for the ribosomal genes of genome $\mathcal{G}_{0}$ and let

$$
P_{0, \text { rib }}(g \mid \pi):=q_{a_{1} c_{1}}^{(0, \mathrm{rib})} \cdot q_{a_{2} c_{2}}^{(0, \mathrm{rib})} \cdot \ldots \cdot q_{a_{3} c_{3}}^{(0, \mathrm{rib})}
$$

If

$$
t_{\mathrm{rib}}(g):=\ln \frac{P_{0, \mathrm{rib}}(g \mid \pi)}{P_{\rho}(g \mid \pi)}>\theta
$$

we consider the gene $g$ as not alien (see $[2,8])$.

The threshold $\theta$ is determined as follows: Let $\mu_{0}$ and $\mu_{0, \text { rib }}$ be the mean values and $\sigma_{0}$ and $\sigma_{0, \text { rib }}$ be the standard deviations of the test statistic $t_{\text {rib }}(G)$, where $G$ is distributed according to $P_{0}(\cdot \mid \pi)$ and $P_{0, \text { rib }}(\cdot \mid \pi)$, respectively. The distribution functions of $1 / \sigma_{0}\left(t_{\text {rib }}(G)-\mu_{0}\right)$ and $1 /$ $\sigma_{0, \text { rib }}\left(t_{\text {rib }}(G)-\mu_{0, \text { rib }}\right)$ are approximately standard normal. We choose $\theta$ in such a way that

$$
1-\Phi\left(\frac{\theta-\mu_{0}}{\sigma_{0}}\right)=\Phi\left(\frac{\theta-\mu_{0, \mathrm{rib}}}{\sigma_{0, \mathrm{rib}}}\right) .
$$

Thus, the error of the first and second kind are of equal size.

\section{Architecture of the HMM}

Figure 1 depicts the architecture of the implemented HMM. The state NATIVE corresponds to genes having an unsuspicious codon usage. The states PUTAL ${ }_{1}, \mathrm{PUTAL}_{2}, \ldots$, PUTAL $_{r}$ represent putatively alien genes originating from taxa $\mathcal{T}_{1}$ to $\mathcal{T}_{r}$. GIs frequently have a mosaic structure which is due to their generation in a multistep process (see [2]). Therefore, we allow transitions from any PUTAL (i.e. donor) state to any other one.

In order to implement our sensitivity controller, we let the transition probabilities depend on the protein under consideration. Thus, the Markov chain presented in Figure 1 is in fact an inhomogeneous one driven by the series $\mathcal{P}_{0}$ of proteins encoded by $\mathcal{G}_{0}$. To simplify notation, we have omitted the index $\pi$, which refers to the protein. Instead, we identify a protein by its index originating from $\mathcal{P}$.

Solving some linear equations, the transition probabilities given in Figure 1 can be determined in such a way that

$a \cdot \tau_{\rho, \alpha}=\frac{p_{\mathrm{gi} 2 \mathrm{gi}, \rho}}{p_{\mathrm{gi} 2 \mathrm{na}}}$ and $b \cdot \tau_{\rho, \alpha}=\frac{p_{\mathrm{na} 2 \mathrm{gi}, \rho}}{p_{\mathrm{na} 2 \mathrm{na}}}$.

$a$ and $b$ are positive constants which were chosen appropriately to generate GIs which are at mean shorter than the surrounding regions of native genes. The probabilities $p_{x 2 y}$ correspond to transitions from state $x$ to $y$ (see Figure 1).

We extend the Markov chain $X_{1}, X_{2}, \ldots, X \ell$ driven by the state diagram given in Figure 1 to a HMM $X_{1}, Y_{1}, X_{2}, Y_{2} \ldots$, $X \ell, Y \ell$ in the following way: For $\pi=1,2 \ldots, \ell$, the random emission $Y_{\pi}$ takes values in the sample space $\mathcal{F}_{\pi}$ defined above. For $\rho=1,2, \ldots, r$, the emission probabilities are defined by means of Equation 1 as follows: 
$P\left(Y_{\pi}=g \mid X_{\pi}=\mathrm{NATIVE}\right)=P_{0}(g \mid \pi) \quad$ and $\quad P\left(Y_{\pi}=g \mid X_{\pi}=\right.$ $\left.\operatorname{PUTAL}_{\rho}\right)=P_{\rho}(g \mid \pi)$.

As already explained, PHX genes have to be eliminated. Our test for putatively highly expressed genes classifies genes as phx or $\neg$ phx. In order to integrate these predictions into the HMM, we interpret the outputs as a random sequence $H_{1}, H_{2}, \ldots, H \ell$ of hints. Please note that an emission is now a combination of a gene and a hint. Hints are interpreted the following way: For the native state we define

$P\left(H_{\pi}=\operatorname{phx} \mid X_{\pi}=\right.$ NATIVE, $\left.Y_{\pi}=g_{\pi}\right)= \begin{cases}1 & \text { if } t_{\text {rib }}\left(g_{\pi}\right)>\theta ; \\ 0 & \text { otherwise }\end{cases}$

For $\rho=1,2, \ldots, r$, the emission probability given a pA state is defined by

$P\left(H_{\pi}=\neg\right.$ phx $\mid X_{\pi} \in\left\{\right.$ PUTAL $_{1}$, PUTAL $_{2}, \ldots$, PUTAL $\left.\left._{r}\right\}\right)=1$.

It is biological evidence, which led to the above definitions. The products of highly expressed genes are involved in complex interactions. Therefore, it is highly unlikely that these genes can be replaced by HGT. Please note that the algorithm has - due to our design - to consider each hint.

\section{Determination of the codon-specific core and atypical genes}

It might be that some pA genes originate from sources not characterized by our set of putative donors (see below). In order to identify these atypical genes, we determine the codon-specific core (CSC) of a genome, which consists of those genes having an unsuspicious codon usage. Having chosen a protein $\pi \in \mathcal{P}_{0}$ and the related gene $g \in \mathcal{G}_{0}$, we consider a random element $G$ of the set $\mathcal{F}_{\pi}$ distributed according to $P_{0}(\cdot \mid \pi)$ (see Equation 1 ). For the following test, we identifed those amino acids $a$ encoded by more than one codon and occurring at least 5 times $\left(n_{a} \geq 5\right)$ in the protein. For each codon $c$ which encodes amino acid $a$ we introduce a random variable $\operatorname{count}_{c}(G)=\# c$, which follows a binomial distribution characterized by the expected value $n_{a} q_{a c}^{(0)}$ and variance $n_{a} q_{a c}^{(0)}\left(1-n_{a} q_{a c}^{(0)}\right)$. The statistic

$$
\phi_{c}(G):=\frac{\operatorname{count}_{c}(G)-n_{a} q_{a c}^{(0)}}{\sqrt{n_{a} q_{a c}^{(0)}\left(1-q_{a c}^{(0)}\right)}}
$$

is approximately distributed according to the standard normal distribution. For each $\delta \in(0,1)$ there is exactly one $\theta_{\delta}>0$ such that

$P\left(\left|\phi_{c}(G)\right| \geq \theta_{\delta}\right)=2 \Phi\left(-\theta_{\delta}\right)=\frac{\delta}{\gamma}$

where $\gamma$ is the occurrence of those amino acids considered in this section. In analogy to [32], we name the gene $g \delta$ typical $(\delta \in(0,1))$, if for all codons $c$

$\left|\phi_{c}(g)\right|<\theta_{\delta}$

This is why the probability of being not $\delta$-typical is for a random gene $\mathrm{G}$ less than or equal to $\delta$. Setting $\delta$ to $10 / \ell$, where $\ell$ is the number of $\mathcal{G}_{0}$ 's genes, turned out to be adequate. Only few genes $(<1 \%)$ were labelled as atypical (see Results). Therefore, the exact value of $\delta$ is uncritical. This observation confirms that our selection of codon usage tables covers the prevalences of putative donors to a great extent.

The algorithm for computing the CSC of genome $\mathcal{G}_{0}$ first removes all genes from $\mathcal{G}_{0}$ that are not $\delta$-typical. Then the synonymous codon frequencies of the remaining genes $\mathcal{G}_{\text {typ }}$ are recomputed and the genes not $\delta$-typical with respect to the new frequencies are removed from $\mathcal{G}_{t y p}$. This is done as long as there are such genes in $\mathcal{G}_{\text {typ }}$. Our experiments showed that this algorithm converged for all completely sequenced genomes to a CSC $\mathcal{G}_{\text {typ }}$ containing at least $75 \%$ of all genes. The atypical genes are those not contained in the CSC $\mathcal{G}_{t y p}$.

\section{Predicting genomic islands}

Using the Viterbi algorithm (see e.g. [33,34]), SIGI-HMM computes at first the Viterbi path (i.e. the most probable sequence of states). All genes labeled as atypical and all genes assigned to one of the states $\operatorname{PUTAL}_{\rho}(\rho=1,2, \ldots, r)$ are considered as belonging to GIs. Since it is reasonable to expect inside GIs genes with a codon usage similar to native ones, GIs separated by less than four native genes can optionally be merged. This merging distance can be set by the user.

\section{Selecting putative donors}

For each genome $\mathcal{G}_{0}$, an individual set of putative donors $\mathcal{G}_{1}, \mathcal{G}_{2}, \ldots, \mathcal{G}_{r}$ has to be selected. As these donors are reduced to their specific codon usage tables, we utilized the Codon Usage Database (CUTG) (Release 149.0, September 26, 2005) [35]. Those entries were extracted that 
consisted of more than 6,400 codons. If a species was represented by more than one table, we took the entry sampling the largest number of codons. This pre-computing phase resulted in the selection of $z=690$ codon usage tables. Then, a $z \times z$ dissimilarity matrix $D$ was set up. For each pair $i, j$ of species, we calculated the value $D_{i j}=1 / 2$ $\eta_{i j}$. In order to compute the discriminative error $\eta_{i j}$, we first considered the set of all "synthetic" genes each comprising 50 codons. Each of the 50 codons was independently selected according to the codon frequencies $q_{c}^{(k)}$. We then determined a probability distribution $P_{k}$ for each species $k$ on this set. These distributions were utilized to determine $\eta_{i j}$ in analogy to Equation 4 .

Hierarchical divisive clustering [36] was now applied to analyze the dissimilarity matrix $D$. As it was our aim to generate clusters representing taxonomically related species, we used the data basis of the taxonomy browser of the NCBI $[37,38]$ for the following procedure. First, we eliminated all entries, which could not be related to a taxonomical class. Then, we generated for the initiation of the diversification process "class"-clusters consisting of species (i.e. synonymous codon frequency tables) belonging to the same taxonomical class. To test homogeneity of the clusters $G$, we computed for each entry $i$ the average dissimilarity $\operatorname{diss}_{G}^{(a v)}(i)$ (see [39]) according to

$$
\operatorname{diss}_{G}^{(a v)}(i):=\frac{1}{|G \backslash\{i\}|} \sum_{j \in G \backslash\{i\}} D_{i j} .
$$

In order to initiate the split of a cluster $G$, the element $i \in$ $G$ having the maximal $\operatorname{diss}_{G}^{(a v)}(i)$ value was chosen. This $i$ was the first element of a new cluster $H$. As long as the condition

$$
\max _{k \in G}\left(\operatorname{diss}_{G}^{(a v)}(k)-\operatorname{diss}_{H}^{(a v)}(k)\right) \geq 0
$$

was true, the element $k$ generating the maximal $\operatorname{diss}_{G}^{(a v)}(k)-\operatorname{diss}_{H}^{(a v)}(k)$ value was transferred from $G$ to $H$. Starting with the initial set of class-clusters described above, the split procedure was applied to that cluster $G$ having maximal diameter

$$
\operatorname{diam} G:=\max _{i, j \in G} D_{i j}
$$

as long as that maximal diameter was greater than or equal to a threshold $d_{1}$ (see [40]).

The procedure resulted in $\tilde{r}=99$ clusters. In order to select a typical example for each cluster, the frequency table having the lowest dissimilarity value to the barycenter of the cluster was chosen. The resulting $\tilde{r}$ codon usage tables were regarded as representatives for putative sources of aliens genes.

To prevent false predictions, clusters with a composition too similar to the input genome $\mathcal{G}_{0}$ have to be eliminated. Therefore, the set of $\tilde{r}$ codon usage tables was preprocessed during the initialization phase for $\mathcal{G}_{0}$. Those elements were deleted, whose dissimilarity to the frequency table of $\mathcal{G}_{0}$ was less than a threshold $d_{2}$. This procedure resulted in a $\mathcal{G}_{0}$-specific set of $r$ putative sources.

\section{Testing performance and analyzing genomes}

To assess accuracy, SIGI-HMM's predictions were compared with results published in [41]. In nearly all cases, the fraction of pA genes determined by SIGI-HMM was lower; compare results listed in Table 1. This might be due to the focusing of SIGI-HMM on the prediction of GIs. However, for the genome of Borrelia burgdorferi SIGIHMM predicts a significantly higher fraction of pA genes. The organization of this genome is unusual, it consists of 20 mainly linear replicons and is subject to frequent genomic rearrangements [42]. During these reorganization events integration of alien DNA might take place making a larger fractions of $\mathrm{pA}$ genes for the $B$. burgdorferi genome plausible. In the following, we report in more detail findings deduced for genomic data sets of the following microbial genomes: Vibrio cholerae, Bacillus subtilis, Escherichia coli K-12, Methanosarcina mazei, Thermus thermophilus and Propionibacterium acnes. The genome of $V$. cholerae consists of two chromosomes with a pronounced asymmetry in the distribution of coding elements with respect to the replicons [43]. Most genes required for growth and virulence are located on chromosome I, whereas chromosome II contains a larger fraction of hypothetical genes.

Interestingly, SIGI-HMM predicted $4.6 \%$ pA genes for chromosome I and $21.1 \%$ pA genes for chromosome II. Two predicted genomic islands on chromosome I comprise a gene cluster for a toxin-coregulated pilus (VC0813 - VC0845) and fragments of a temperate filamentous phage (VC1455 - VC1457, VC1464, VC1477 - VC1481). Both clusters are closely associated with the pathogenicity of $V$. cholerae [44]. Many of the hypothetical genes encoded on chromosome II are located within a large integron island comprising gene products that might be 
involved in drug resistance, DNA metabolism and virulence [43]. One of the predicted GIs on chromosome II, which consists of genes VCA0283 - VCA0507, overlaps to a great extent the integron described above. SIGI-HMM identified two additional GIs comprising genes VCA0198 - VCA0202 and VCA0790 - VCA0797, which contain homologs for putative transposases. As transposases are often encoded in genetically mobile IS-elements, these genes are likely candidates for alien genes. For both chromosomes, SIGI-HMM predicts similar distributions of putative donors. The largest fractions belong to the class of bacilli (51\% or $61 \%$ ), whereas the taxonomical class of $V$. cholerae, the $\gamma$-proteobacteria, accounts for $34 \%$ or $37 \%$ of all pA genes.

For B. subtilis, 10 integrated prophages have been reported (see $[4,45,46]$, and [47]), whose identification is based either on experimental evidence or theoretical considerations. A profound analysis of chromosomal heterogeneities has been accomplished by Nicolas et al. [9], using a HMM on the nucleotide level. All genomic islands identified by Nicolas et al. were largely confirmed by SIGIHMM. Both approaches detected nine of the putative prophages and several other islands assigned to functions in cell wall biosynthesis, competence and resistance. In contrast to Nicolas et al., SIGI-HMM identified pA genes, which belong to the experimentally reported integrated prophage PBSX [47]. In summary, SIGI-HMM predicted for $B$. subtilis $9.5 \%$ of the genes as being $\mathrm{pA}$, most of them originating from the class of bacilli (316 pA genes, $81 \%$ ).

Based on a combination of parameters measuring computational complexity, Lawrence and Ochman [4] had estimated that about $18 \%$ of the E. coli $\mathrm{K}-12$ genome have been imported via lateral gene transfer. In contrast, SIGIHMM predicted 580 (13.4\%) pA genes which were mostly organized in small clusters of less than ten genes. $521 \mathrm{pA}$ genes $(92 \%)$ seem to originate from $\gamma$-proteobacteria, the taxonomical class E. coli belongs to. The largest GIs included the cryptic prophages CP4-6 (262 - $297 \mathrm{kbp})$, DLP12 (557 - $584 \mathrm{kbp}), \mathrm{e} 14(1,196$ - 1,221), Rac $(1,410$ - 1,433 kbp), Qin (1,631 - 1,651 kbp), CP4-44 (2,064 $2,069 \mathrm{kbp})$, CPS-53 (2,465 - 2,475 kbp), Eut $(2,556-$ $2,563 \mathrm{kbp}), \mathrm{CP} 4-57$ (2,752 - 2,775 kbp), and the phagelike element KpLE2 (4,494 - 4,544 kbp) (for review see [48]). 44 IS-elements have been annotated within the genome of E. coli K-12, SIGI-HMM predicted 34 of them correctly.

T. thermophilus is an extreme thermophilic bacterium living as a halotolerant in an extreme ecological niche. Two T. thermophilus strains, namely HB27 [45] and HB8 [46], have been sequenced so far. SIGI-HMM predicted for both strains a small fraction of pA genes (HB27 1.0\%; HB8 $1.7 \%)$. The largest pA cluster consists of 6 genes in case of
HB27 (TTC0277 - TTC0278, TTC0280 - TTC0283) and of 5 genes in case of HB8 (TTHA0644 - TTHA0648). The GIs share no sequence similarity and contain genes that are associated with functions in cell wall biosynthesis. Most pA genes seem to originate from the class of the $\delta$-proteobacteria (HB27 5 genes; HB8 18 genes). In both genomes no donor was predicted for $12 \mathrm{pA}$ genes, respectively.

It has been suggested that HGT plays an important role in the evolution of the mesophilic archaeon M. mazei [49]. The analysis of protein sequences via BLAST showed that $31 \%$ of the archeal sequences were more similar to bacterial than to archeal ones. SIGI-HMM predicted for $M$. mazei only $8.4 \%$ pA genes. Please note that the two analyses used different approaches for $\mathrm{pA}$ prediction and that SIGI-HMM focuses on the analysis of GIs only. These systematic differences may explain the findings. Interestingly and in agreement with the above analysis, only $21 \%$ of the pA genes seem to originate from the archeal domain. $27 \%$ of the pA genes were predicted to originate from the class of shingobacteria, $23 \%$ from chlamydia and $11 \%$ from clostridia. This finding is also in agreement with the postulated gene flux from mesophilic bacteria to mesophilic archaea [27].

P. acnes is a major inhabitant of the adult human skin, living in sebaceous follicles [50]. Usually the bacterium is harmless; however it is involved in acne vulgaris formation. The genome harbors genes whose products are involved in degrading host molecules and pore-forming factors. It also contains surface-associated and other immunogenic factors, which might be responsible for acne inanimation and other $P$. acnes-associated diseases. SIGI-HMM predicted $4.1 \%$ pA genes clustered in five larger GIs and several smaller islands of less than five genes. $47 \%$ ( 45 genes) of them are predicted to originate from the $\alpha$-proteobacteria, but only 13\% (12 pA genes) from the taxonomic class of $P$. acnes, the actinobacteria. Interestingly, four of the larger GIs and two of the smaller islands are flanked by tRNA-genes in direct or close vicinity. tRNAs are considered to be hot spots for recombination events that can result in horizontal gene transfer. SIGI-HMM found these anomalies although it does not interpret sequences besides protein coding genes. Of the larger GIs, the first (at position $28-34 \mathrm{kbp}$ ) contains genes without functional assignment, the second (874 $880 \mathrm{kbp})$ harbors genes for several transport systems among others for iron(III)dicitrate (PPA0792 - PPA0794) and the third (921 - $941 \mathrm{kbp})$ for an ABC-type transport system (PPA0843 - PPA0845), putative conjugal transfer proteins (PPA0846 - PPA0848) and two putative transposases (PPA2354, PPA0858). The forth GI $(1,390$ $1,407 \mathrm{kbp})$ contains a gene cluster for a putative nonribosomal peptide synthetase (NRPS) (PPA1287 PPA1290). NRPSs are involved in the biosynthesis of 


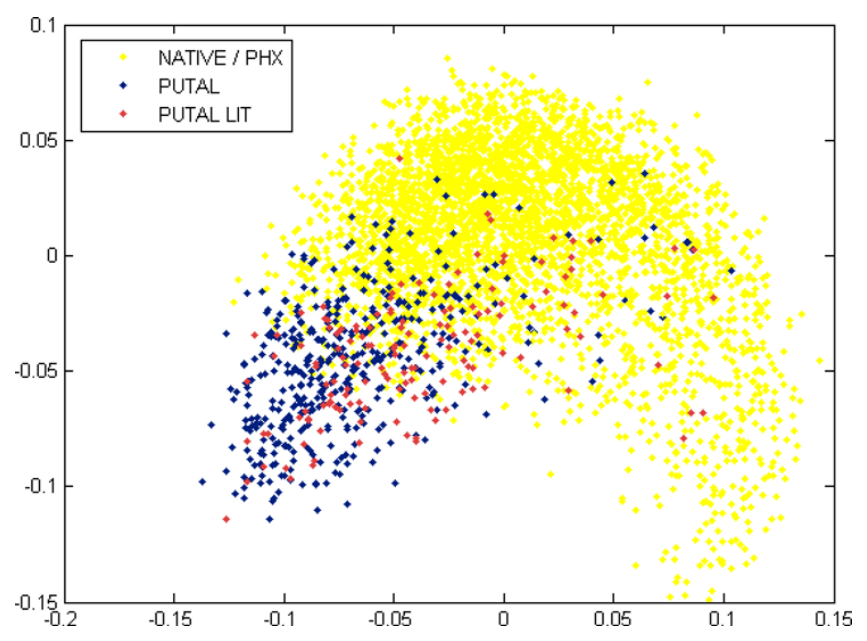

Figure 2

Kernel-based scatter plot visualization of SIGI-HMM predictions for $E$. coli $\mathrm{K}-12$. Blue points (PUTAL) represent $\mathrm{PA}$ genes as predicted by SIGI-HMM, red points (PUTAL LIT) indicate predicted $p A$ genes with additional evidence from the current literature as described in the text. Yellow points (NATIVE / PHX) refer to genes which are predicted to be native or highly expressed.

complex secondary metabolites. As many of the genes clustered in the fifth GI $(1,707-1,731 \mathrm{kbp})$ are annotated as phage-associated proteins (PPA1593 - PPA1596, PPA1604 - PPA1605), the GI may be attributed to an integrated prophage.

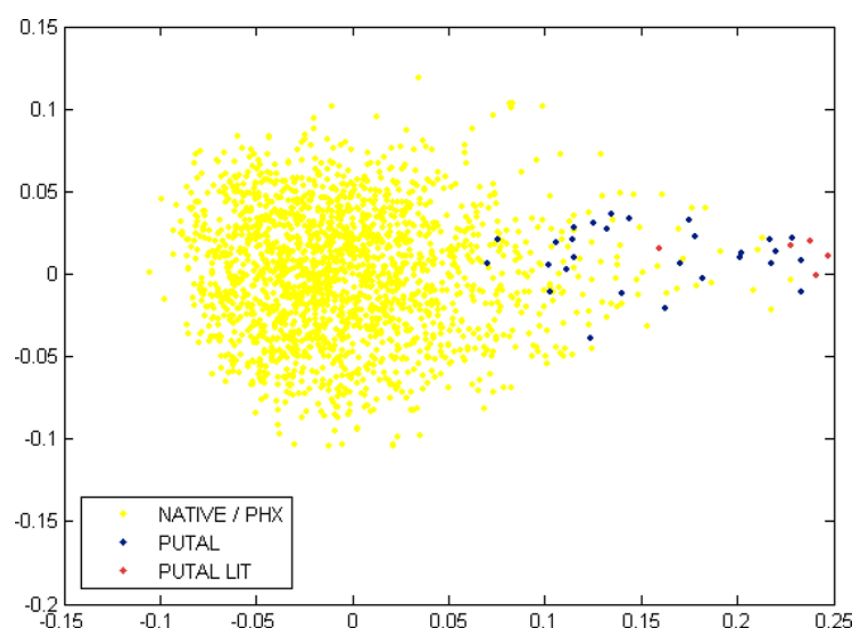

Figure 3

Kernel-based scatter plot visualization of SIGI-HMM predictions for T. thermophilus. Blue points (PUTAL) represent $\mathrm{PA}$ genes as predicted by SIGI-HMM, red points (PUTAL LIT) indicate predicted $\mathrm{pA}$ genes with additional evidence from the current literature as described in the text. Yellow points (NATIVE / PHX) refer to genes which are predicted to be native or highly expressed.

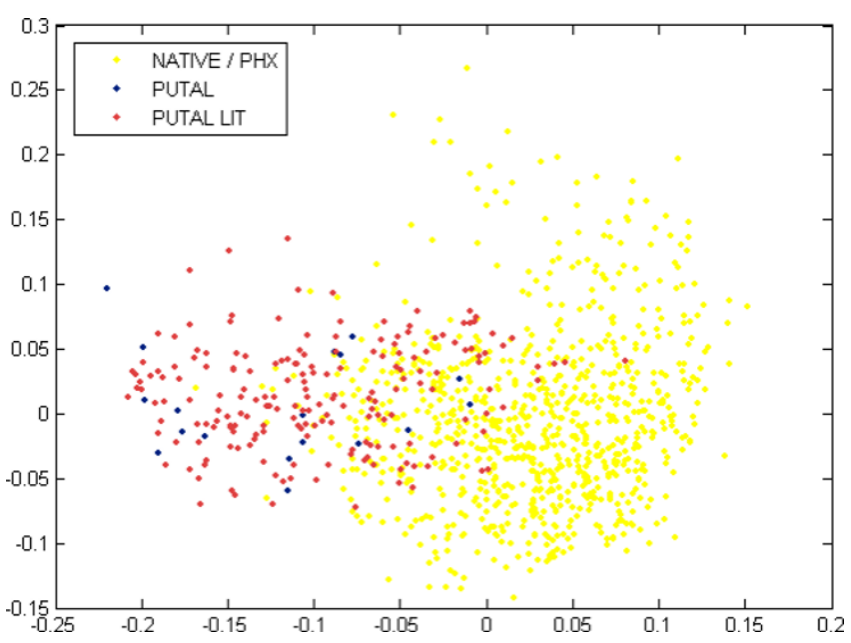

Figure 4

Kernel-based scatter plot visualization of SIGI-HMM predictions for V. cholerae (chromosome II). Blue points (PUTAL) represent $P A$ genes as predicted by SIGI-HMM, red points (PUTAL LIT) indicate predicted $p A$ genes with additional evidence from the current literature as described in the text. Yellow points (NATIVE / PHX) refer to genes which are predicted to be native or highly expressed.

For visualization of the HMM-based predictions we use scatter plot repesentations providing an overview of codon usage similarities between all genes of a genome. By means of a newly developed kernel for measuring similarity of codon usage tables [51], we perform a kernel principal component analysis (see e.g. [52]) to compute the resulting $2 \mathrm{D}$ coordinates of all genes. In that representation, nearby points indicate a similar codon usage of the corresponding genes. It is important to note that the kernel-based approach does not use any information about the location of genes on the genome. Instead, codon usage correlations between different amino acids are used to derive the two-dimensional representation. This approach is different from the concept of SIGI-HMM. Therefore, a clustering of SIGI-HMM predicted pA genes which becomes visible in the scatter plots (see Figure 2, 3, and 4) confirms the corresponding predictions.

Figure 2 is a plot of all genes of the E. coli K-12 genome. The general form resembles the "rabbit head" trimodal shape described earlier for the genome of B. subtilis [53]. Most genes belonging to integrated prophages are located in the lower left "ear". PHX genes are clustered in the lower right corner.

T. thermophilus is one of the genomes with lowest pA content. The plot depicted in Figure 3 represents the genome of T. thermophilus and has a quite specific shape. This finding indicates that the overall shape of the plot is massively modulated by the fraction of genes acquired via HGT. The 
Table 2: Prophages and prophage-like elements integrated into the genome of $B$. subtilis. Column I lists the elements flanked by sequence repeats. Column 2 gives the location of the repeats. Column 3 and 4 list the number of $\mathrm{pA}$ and $\mathrm{pN}$ genes predicted for these GIs by SIGI-HMM. The two last columns indicate the offset of the GI from the sequence repeats. An offset of $-I$ means that the GI predicted by SIGI-HMM starts (ends) one gene after (before) the repeat. Positions are as in [9] and given in kbp.

\begin{tabular}{lccccc}
\hline Element & Repeats & \# pA & \# pN & Offset Begin & Offset End \\
\hline PI & $202-213$ & 10 & I & 0 & -1 \\
P2 & $555-567$ & 10 & 1 & -1 & 0 \\
P6 & $2050-2060$ & 9 & 0 & 0 & 0 \\
Skin & $2654-2701$ & 32 & 31 & 0 & 0 \\
P7 & $2725-2735$ & 7 & 6 & -6 & 0 \\
\hline
\end{tabular}

pA genes as predicted by SIGI-HMM are mainly located in a long tail with low point density on the right hand side of the plot.

As already mentioned, the genome of $V$. cholerae consists of two chromosomes. Most essential genes are located on chromosome I and codon usage of genes on chromosome II is rather inhomogeneous. Again, the overall shape of the plot, which represents chromosome II, reflects this situation (compare Figure 4) and shows a well-clustering fraction of pA genes located in the lower left corner of the plot. Please note that the positioning of pA genes predicted by SIGI-HMM only and those pA genes supported by additional evidence from the literature corresponds to a great extent in all plots.

\section{Assessing the patchiness of Gls}

Genomic islands are thought to be the result of constant genetic rearrangement events, which account for their observed mosaic structure. As these rearrangements could also take place at hot spots for the integration of alien DNA in the host genome, patches of genes having a codon usage similar to the host have to be expected inside GIs. This fact makes it difficult to determine the number of false negatives, even in annotated GIs. The number of false positives is difficult to deduce too, as it is hard to proof that a stretch of pA genes has not been acquired via HGT. In order to illustrate the problem and the patchiness of GIs, we compare in more detail some predictions with published findings.

Chromosome II of $V$. cholerae contains an integron island of size $125.3 \mathrm{kbp}$, which includes genes VCA0271 to VCA0491 [43]. Of these 214 genes, SIGI-HMM labels 188 as $\mathrm{pA}(87 \%), 1$ as AT (atypical) and 25 as pN (putatively native). SIGI-HMM did subdivide the integron island into the following patches: VCA0271 - VCA0282 pN, VCA0283 - VCA0286 pA, VCA0287 - VCA0291 pN, VCA0292 - VCA0324 pA, VCA0325 - VCA0329 pN, VCA0330 - VCA0379 pA, VCA0380 - VCA0385 pN,
VCA0386 - VCA0507 pA. From the remaining 611 genes on the chromosome, 42 were predicted as $\mathrm{pA}$.

The chromosome of Mesorhizobium loti consists of 6.725 protein coding genes. It contains a $611 \mathrm{kbp}$ DNA segment which is, as the authors put it, "a highly probable candidate of a symbiotic island" [3]. SIGI-HMM predicted 5.561 genes as $\mathrm{pN}, 1.161(17 \%)$ as $\mathrm{pA}$ and 30 as AT. Of the symbiotic island, 145 genes were $\mathrm{pN}, 421 \mathrm{pA}(72 \%)$ and 14 AT. The pA genes were clustered in 29 GIs ranging in size from 2 to 108 genes.

As already mentioned, ten integrated prophages or prophage-like elements were reported for the genome of $B$. subtilis [9]. Five of these elements are flanked by sequence repeats which we considered as the original integrations sites indicating the actual borders of the GIs. Table 2 summarizes composition and location of related GIs predicted by SIGI-HMM. Skin prophage and P7 have a mosaic structure and harbour $\approx 50 \% \mathrm{pN}$ genes. In four of the five cases, the borders of the predicted GIs are in good agreement with the location of the repeats.

\section{Discussion}

\section{Analysis of codon usage reliably allows to identify most} HGT events

We have to stress that our approach entirely relies on the analysis of codon usage. SIGI-HMM does not interpret additional signals like direct repeats or disrupted tRNA sequences frequently flanking GIs. Therefore, the outcome of the HMM analysis are DNA regions showing atypical codon usage. This fact has two consequences: 1 ) SIGI-HMM is unable to identify GIs having an unsuspicious codon usage and 2) the rationale of naming these stretches GIs merely depends on the correlation with biological findings.

However, we have shown that DNA regions identified by SIGI-HMM as suspicious correspond to known cases of horizontally transferred elements like phages. Our approach of focusing on the analysis of codon usage is not a completely new one. There exist several methods to identify horizontally transferred genes. These approaches rely on the analysis of codon or amino acid sequences or the construction of phylogenetic trees. For a comparison see e.g. [14]. Each approach has individual drawbacks and it might be that each method identifies a specific class of genes acquired in a different time of genome evolution [13]. It was argued that codon usage is no reliable indicator for the study of HGT [54]. However, it was shown that related methods identify pA genes to a great extent [55]. The assumption that methods analyzing codon usage might overlook horizontally acquired genes could be valid for more ancient events. For these genes, the effect of amelioration [56] might have rendered codon usage 
unsuspicious. Lawrence and Ochman estimated the age of imported genes [4]. Their conclusion was that most were relatively recent, i.e. acquired within the last few million years; see also [57]. This suggests that older imports have been purged presumably because the acquired genes did not improve fitness. If this argument is true, there is no need to search for larger amounts of ancient pA genes. Therefore, methods based on the analysis of codon usage should have the potential of identifying a great fraction of horizontally transferred genes. Low values of $\mathrm{pA}$ content can frequently be explained with biological findings. It was argued that species populating extreme ecological niches tend to have relative small genomes [58]. The size of the sequenced T. thermophilus genomes support this notion. If selective pressure minimizes genome size, it will also effect acquisition and conservation of foreign DNA. The low fraction of pA genes determined for both strains is in agreement with the above hypothesis.

The methods will fail at alien genes having a codon usage undistinguishable from the host's preferences. Among them might be ancient pA genes. Because of the amelioration process, ancient pA genes are harder to detect. These pA genes, surviving the selection process may actually constitute important and useful genes. In order to complete the set of identified HGT events and to reduce the number of false negatives, it will be necessary to use a completely different approach like the construction of phylogenetic trees.

If not processed correctly, highly expressed genes could be a source for false positive predictions. It is known that these genes show a distinct codon usage by preferring a species-specific set of major codons. In order to reduce the rate of false positive predictions, we use a filter which is based on a method [30] shown to be effective in predicting gene expressivity [31]. We have adjusted the parameters (see Equation 4) in such a way that the errors of the first and second kind are equally likely. Highly expressed genes belong to the core of a genome and it is unlikely that these genes are subject to HGT. Nevertheless, the user may disable this filter in order to study its influence on GI prediction.

\section{Focusing on the prediction of Gls is biologically reasonable and reduces the risk of false predictions}

Intrinsically, increasing the sensitivity of a test also increases the risk of predicting false positives. For the prediction of pA genes, the risk can however be minimized, if an algorithm focuses on the prediction of genomic islands as SIGI-HMM does. The pieces of DNA acquired via HGT typically have a considerable length. Examples are the symbiotic island of size $611 \mathrm{kbp}$ described for the genome of $M$. loti or the integron island of size $125 \mathrm{kbp}$ found on chromosome II of V. cholerae (see Results). Genes respon- sible for pathogenicity are also agglomerated in islands; see [2] and references therein. Therefore, a focusing on predicting GIs rather than all $\mathrm{pA}$ genes is an appropriate strategy to avoid false positives without missing relevant HGT events. Consequently, this argument was considered for the design of recently introduced algorithms $[23,59]$. However, the rate of false positive predictions will increase, if codon usage of a genome is inhomogeneous. To avoid this situation, it is important, to determine the CSC of a genome.

\section{Codon usage is a reliable indicator to predict the origin of pA genes}

For each completely sequenced genome, we have computed a variant of the CSC defined above; see [60]. It consisted of those genes having a homogeneous codon usage. The results obtained with the classification of genes from CSCs show that codon usage hints at the origin of genes. First tests indicate that prediction quality is high, as long as the CSC contains at least $70 \%$ of the genes. In addition, the results of performance tests (see [23]) carried out to demonstrate SIGI's ability of predicting the putative donor are also valid for SIGI-HMM.

Omelchenko et al. [61] used BLAST on the protein level to determine HGT events in the genome of T. thermophilus HB27. The protein sequences of many genes were similar to those of hyperthermophilic archaea. Taxonomical classification of donors for genes constituting GIs predicted by SIGI-HMM was rather inhomogeneous. The putative donors belonged to bacteria, archaea and eukaryota. It will be necessary to evaluate methods for pA prediction with a standardized test bed. Artificial genomes as introduced recently [62] may constitute the basis for such a validation, which may lead to a contest of methods for $\mathrm{pA}$ prediction.

\section{Conclusion}

An inhomogeneous HMM on gene level allows to identify GIs in microbial genomes and to predict the putative donor of horizontally transferred genes. The predictions are consistent with known findings and do not depend on the optimization of many parameters. Our implementation as a freely available tool written in Java allows an independent inspection of genomes in great detail. The genome-specific predictions can be used for further analysis or the comparison of several methods.

\section{Authors' contributions}

SW and RM specified the problem and the solution strategy. SW developed the HMM together with OK and KS and provided resources. $\mathrm{OK}$ and $\mathrm{TB}$ implemented the HMM. The donor selection was conceptualized by SW, RA and $\mathrm{CD}$, and implemented by RA. WFF analyzed the genomes. PM decisively contributed to the methods meas- 
uring similarity of codon usage tables. RM conducted the performance tests and contributed substantially to the manuscript which was prepared together with SW and KS. All authors read and approved the final manuscript.

\section{Acknowledgements}

The research was partially supported by the grant "ELAN - E-Learning Academic Network" of the Lower Saxony Ministry of Science, and by DFG Graduate Program "Identification in mathematical models: Synergy of stochastics and numerical methods".

\section{References}

I. Gogarten J, Doolittle W, Lawrence J: Prokaryotic evolution in light of gene transfer. Mol Biol Evol 2002, 19:2226-2238.

2. Hacker J, Kaper JB: Pathogenicity islands and the evolution of microbes. Annu Rev Microbiol 2000, 54:64I-679.

3. Kaneko T, Nakamura $Y$, Sato S, Asamizu E, Kato T, Sasamoto $S$, Watanabe A, Idesawa K, Ishikawa A, Kawashima K, Kimura T, Kishida Y, Kiyokawa C, Kohara M, Matsumoto M, Matsuno A, Mochizuki Y, Nakayama S, Nakazaki N, Shimpo S, Sugimoto M, Takeuchi C, Yamada $M$, Tabata S: Complete genome structure of the nitrogen-fixing symbiotic bacterium Mesorhizobium loti. DNA Res 2000, 7:38I-406.

4. Lawrence JG, Ochman H: Molecular archaeology of the Echerichia coli genome. Proc Nat Acad Sci USA 1998, 95:94I3-94I7.

5. Hooper SD, Berg OG: Detection of genes with atypical nucleotide sequence in microbial genomes. J Mol Evol 2002, 54:365-375

6. Mrázek J, Karlin S: Detecting alien genes in bacterial genomes. Ann NY Acad Sci 1999, 870:314-329.

7. Garcia-Vallvé S, Romeu A, Palau J: Horizontal gene transfer in bacterial and archaeal complete genomes. Genome Res 2000, 10:1719-1725

8. Karlin S: Detecting anomalous gene clusters and pathogenicity islands in diverse bacterial genomes. Trends Microbiol 200I, 9(7):335-343. Jul

9. Nicola P, Bize L, Muri F, Hoebeke M, Rodolhe F, Ehrlic SD, Prum B, Bessièrs $\mathrm{P}$ : Mining Bacillus subtilis chromosome heterogeneities using hidden Markov models. Nucleic Acids Res 2002, 30:1418-1426.

10. Nesbø CL, L'Haridon S, Stetter KO, Doolittle WF: Phylogenetic analysis of two "archaeal" genes in Thermotoga maritima reveal multiple transfers between archaea and bacteria. Mol Biol Evol 200I, 18:362-375.

II. Sandberg R, Winberg G, Bräden C, Kaske A, Ernberg I, Cöster J: Capturing whole-genome characteristics in short sequences using a naive Bayesian classifier. Genome Res 200I, I I:1404-1409.

12. Dufraigne C, Fertil B, Lespinats S, Giron A, Deschavanne P: Detection and characterization of horizontal transfers in prokaryotes using genomic signature. Nucleic Acids Res 2005, 33:e6.

13. Ragan MA: Detection of lateral gene transfer among microbial genomes. Curr Opin Genet Dev 200I, I I:620-626.

14. Ragan MA: On surrogate methods for detecting lateral gene tranfer. FEMS Microbiol Lett 200I, 201:187-191.

15. Grantham R, Gautier C, Gouy M, Mercier R, Pave A: Codon catalog usage and the genome hypothesis. Nucleic Acids Res 1980, 8:R49-R62.

16. Burge $C$ : Identification of genes in a human genome DNA. In PhD thesis Stanford University; 1997.

17. Burge C, Karlin S: Prediction of complete gene structures in human genomic DNA. J Mol Biol 1997, 268:78-94.

18. Krogh A: Two methods for improving performance of an HMM and their application for gene finding. Proc Int Conf Intell Syst Mol Biol 1997, 5: 179-186.

19. Krogh A: Using data base matches with HMMGene for automated gene detection in Drosophila. Genome Res 2000, 10:523-528.

20. Yeh R, Lim L, Burge C: Computational inference of homologous gene structures in the human genome. Genome Res 200I, II:803-816.
21. Stanke M, Waack S: Gene prediction with a hidden Markov model and new intron submodel. Bioinformatics 2003, 19:ii2। 5 -ii225.

22. Stanke M, Schöffman O, Dahms S, Morgenstern B, Waack S: Gene prediction in eukaryotes with a generalized hidden Markov model that uses hints from external sources. BMC Bioinformatics 2006, 7:62.

23. MerkI R: SIGI: score-based identification of genomic islands. BMC Bioinformatics 2004, 5:22.

24. Collins N, Liebenberg J, de Villiers E, Brayton K, Louw E, Pretorius A, Faber F, van Heerden $H$, Josemans A, van Kleef $M$, Steyn $H$, van Strijp $M$, Zweygarth E, Jongejan F, Maillard J, Berthier D, Botha M, Joubert F, Corton C, Thomson N, Allsopp M, Allsopp B: The genome of the heartwater agent Ehrlichia ruminantium contains multiple tandem repeats of actively variable copy number. Proc Natl Acad Sci USA 2005, 102:838-843.

25. Veith B, Herzberg C, Steckel S, Feesche J, Maurer K, Ehrenreich P, Baumer S, Henne A, Liesegang H, Merkl R, Ehrenreich A, Gottschalk $G$ : The complete genome sequence of Bacillus licheniformis DSMI3, an organism with great industrial potential. J Mol Microbiol Biotechnol 2004, 7:204-2II.

26. Merkl R: A comparative categorization of protein function encoded in bacterial or archeal genomic islands. I Mol Evol 2006, 62: I- 14.

27. Wiezer A, Merkl R: A comparative categorization of gene flux in diverse microbial species. Genomics 2005, 86:462-475.

28. Colombo homepage [http://www.tcs.informatik.uni-goettin
gen.de/colombo]

29. Rutherford K, Parkhill J, Crook J, Horsnell T, Rice P, Rajandream MA Barrell B: Artemis: sequence visualisation and annotation. Bioinformatics 2000, 16:944-945.

30. Merkl R: A survey of codon and amino acid frequency bias in microbial genomes focusing on translational efficiency. J Mol Evol 2003, 57:453-466.

31. Supek F, Vlahovicek K: Comparison of codon usage measures and their applicability in prediction of microbial gene expressivity. BMC Bioinformatics 2005, 6: 182.

32. Welsh D: Codes and Cryptograpy New York: Oxford University Press; 1987.

33. Durbin R, Eddy S, Krogh A, Mitchinson G: Biological Sequence Analysis Cambridge: Cambridge University Press; 1998.

34. Merkl R, Waack S: Bioinformatik interaktiv - Algorithmen und Praxis Weinheim: Wiley-VCH; 2003.

35. Nakamura $Y$, Gojobori T, Ikemura T: Codon usage tabulated from the international DNA sequences databases and predictions. Nucleic Acids Res 1999, 27:292.

36. Hastie T, Tibshirani R, Friedman J: The Elements of Statistical Learning New York, Berlin, Heidelberg: Springer; 200I.

37. Wheeler D, Chappey C, Lash A, Leipe DD, Madden T, Schuler G, Tatusova T, Rapp B: Database resources of the National Center for Biotechnology Information. Nucleic Acids Res 2000, 28: I0-I4.

38. Benson DA, Karsch-Mizrachi I, Lipman DJ, Ostell J, Rapp BA, Wheeler DL: GenBank. Nucleic Acids Res 2000, 28:15-18.

39. MacNaughton-Smith P, Williams W, Dale M, Mockett L: Dissimilarity analysis: a new technic of hierarchical subdivision. Nature 1964, 202:1034-1035.

40. Kaufman L, Rousseeuw P: Finding Groups in Data New York: Wiley; 1990.

4I. Ochman H, Lawrence JG, Groisman EA: Lateral gene transfer and the nature of bacterial innovation. Nature 2000, 405:299-304.

42. Chaconas G: Hairpin telomeres and genome plasticity in Borrelia: all mixed up in the end. Mol Microbiol 2005, 58:625-635.

43. Heidelberg JF, Eisen JA, Nelson WC, Clayton RA, Gwinn ML, Dodson RJ, Haft DH, Hickey EK, Peterson JD, Umayam L, Gill SR, Nelson KE, Read TD, Tettelin H, Richardson D, Ermolaeva MD, Vamathevan J, Bass S, Qin H, Dragoi I, Sellers P, McDonald L, Utterback T, Fleishmann RD, Nierman WC, White O, Salzberg SL, Smith HO, Colwell RR, Mekalanos JJ, Venter JC, Fraser CM: DNA sequence of both chromosomes of the cholera pathogen Vibrio cholerae. Nature 2000, 406:477-483.

44. Waldor M, Mekalanos J: Lysogenic conversion by a filamentous phage encoding cholera toxin. Science 1996, 272:1910-1914.

45. Kunst F, Ogasawara N, Moszer I, Albertini A, Alloni G, Azevedo V, Bertero M, Bessieres P, Bolotin A, Borchert S, Borriss R, Boursier L, Brans A, Braun M, Brignell S, S B, Brouillet S, Bruschi C, Caldwell B, Capuano V, Carter N, Choi S, Codani J, Connerton I, Danchin A, et 
al:: The complete genome sequence of the gram-positive bacterium Bacillus subtilis. Nature 1997, 390:249-256.

46. Takemaru K, Mizuno M, Sato T, Takeuchi M, Kobayashi Y: Complete nucleotide sequence of a skin element excised by DNA rearrangement during sporulation in Bacillus subtilis. Microbiology 1995, 141:323-327.

47. Wood HE, Dawson MT, Devine K, McConnell D: Characterization of PBSX, a defective prophage of Bacillus subtilis. J Bacteriol 1990, 172:2667-2674

48. Casjens S: Prophages and bacterial genomics: what have we learned so far? Mol Microbiol 2003, 49:277-300.

49. Deppenmeier U, Johann A, Hartsch T, Merkl R, Schmitz R, MartinezArias R, Henne A, Wiezer A, Bäumer S, Jacobi C, Brüggemann $H$, Lienard T, Christmann A, Bömecke M, Steckel S, Bhattacharyya A, Lykidis A, Overbeck R, Klenk HP, Gunsalus RP, Fritz HJ, Gottschalk $\mathrm{G}$ : The genome of Methanosarcina mazei: evidence for lateral gene transfer between archaea and bacteria. J Mol Microbiol Biotechnol 2002, 4:453-46I.

50. Brüggemann $H$, Henne A, Hoster F, Liesegang $H$, Wiezer A, Strittmatter A, Hujer S, Dürre P, Gottschalk G: The complete genome sequence of Propionibacterium acnes, a commensal of human skin. Science 2004, 305:67I-673.

5I. Meinicke P, Brodag T, Fricke WF, Waack S: Kernel-based visualization of codon usage data. . Submitted

52. Schölkopf B, Smola AJ, Müller KR: Nonlinear component analysis as a kernel eigenvalue problem. Neural Computation 1998, 10:1299-1319.

53. Moszer I, Rocha E, Danchin A: Codon usage and lateral gene transfer in Bacillus subtilis. Curr Opin Microbiol 1999, 2:524-8.

54. Wang B: Limitations of compositional approach to identifying horizontally transferred genes. J Mol Evol 200I, 53:244-250.

55. Daubin V, Perrière G: G+C3 structuring along the genome: a common feature in prokaryotes. Mol Biol Evol 2003, 20:47I-483.

56. Lawrence JG, Ochman H: Amelioration of bacterial genomes: rates of change and exchange. J Mol Evol 1997, 44:383-397.

57. de la Cruz F, Davies J: Horizontal gene transfer and the origin of species: lessons from bacteria. Trends Microbiol 2000 , 8:128-133.

58. Bentley S, Parkhill J: Comparative genomic structure of prokaryotes. Annu Rev Genet 2004, 38:77I-792.

59. Nakamura $Y$, Itoh $T$, Matsuda $H$, Gojobori $T$ : Biased biological functions of horizontally transferred genes in prokaryotic genomes. Nat Genet 2004, 36:760-766.

60. Waack S, Brodag T, Surovcik K, MerkI R: Assessing homogeneity and species-specifity of codon usage in prokaryotic genomes. . submitted

6I. Omelchenko M, Wolf Y, Gaidamakova E, Matrosova V, Vasilenko A, Zhai M, Daly M, Koonin E, Makarova K: Comparative genomics of Thermus thermophilus and Deinococcus radiodurans: divergent routes of adaptation to thermophily and radiation resistance. BMC Evol Biol 2005, 5:

62. Azad R, Lawrence J: Use of artificial genomes in assessing methods for atypical gene detection. PLoS Comput Biol 2005, I:e56.

\section{Publish with Biomed Central and every scientist can read your work free of charge}

"BioMed Central will be the most significant development for disseminating the results of biomedical research in our lifetime. "

Sir Paul Nurse, Cancer Research UK

Your research papers will be:

- available free of charge to the entire biomedical community

- peer reviewed and published immediately upon acceptance

- cited in PubMed and archived on PubMed Central

- yours - you keep the copyright
BiolMedcentral 